\title{
Effect of Geometry Design on Fluid Flow, Power Consumption and Strain Rate of Rotor-Stator Mixer
}

\author{
Alexander Kolomiets, Tomáš Jirout, Jan Skočilas \\ Czech Technical University in Prague \\ Technická 4, Prague, Czech Republic \\ Alexander.Kolomiets@fs.cvut.cz; Tomas.Jirout@fs.cvut.cz; Jan.Skocilas@fs.cvut.cz
}

\begin{abstract}
In this paper the influence of design of rotor-stator on its characteristics has been investigated. The drop breakup in laminar flow is depended on combination of elongation in the channel between rotor-stator blades and shear by segment's edges. For investigation of influence of the combination two geometries were created. The first geometry includes long blades on the rotor and stator parts while the second geometry has two times more but also two times shorter blades. Fluid flow and power consumption were measured in CFD simulation of two geometries of rotor-stator. Simulations were completed for laminar regime with non-Newtonian liquid.
\end{abstract}

Keywords: Mixing, CFD, Rotor-stator, Non-Newtonian liquid, Laminar flow.

\section{Introduction}

Rotor-stator mixers are widely used in pharmacy, food, chemical and other industries [1]. However, there is a small amount of information describing characteristics of these devices. Most research works use a water-silicon oil as liquidliquid dispersion. These dispersions are almost inviscid which leads to turbulent regime investigation only. Rotor-stator mixers have a great variety if designs distinguished by blade form, number of slots, number of rows etc. These devices are produced for very special conditions and materials by trial and error, so there is almost lack of information about influence of design on performance. Several research work [2],[3] and [4], were focused on the influence of geometry on the performance of rotor-stator devices.

There is also almost lack of information about behaviour of non-Newtonian liquids and dispersions in such mixers. Some of these liquids has a very high apparent viscosity which can lead to laminar regime of mixing. Under laminar regime of flow the drop breakup has different mechanism then in turbulent. One of the most possibly mechanism of drop creation is combination of shear and elongation for liquid layer deformation [5]. Elongation proceeds along blades of rotor and stator while shear occurs on the edge of these segments. It is still a question which one of this two forces has a greater impact on drop breakup and drop size distribution. For investigation of these phenomena two similar geometries were created.

\section{Materials and geometry}

\section{1. Geometry}

Two geometries were created for the research. Both geometries have rotor and stator of the same diameter, clearance between rotor and stator, two rows of blades on rotor and stator and cross-section of the segments. The difference is in number of segments/slots. The first geometry has a five-segment rotor and six-segment stator (Fig. 1a,b). The second geometry has a ten-segment rotor and twelve-segment stator (Fig. $1 \mathrm{c}, \mathrm{d}$ ). As it is seen, the second geometry has two times more segments/slots, however, these segments/slots have a half of length of the segment/slot of the first geometry, which means that whole round contact length between rotor and stator segments remains the same. Both geometries were simplified for simulation (no shaft, bearings etc.) 


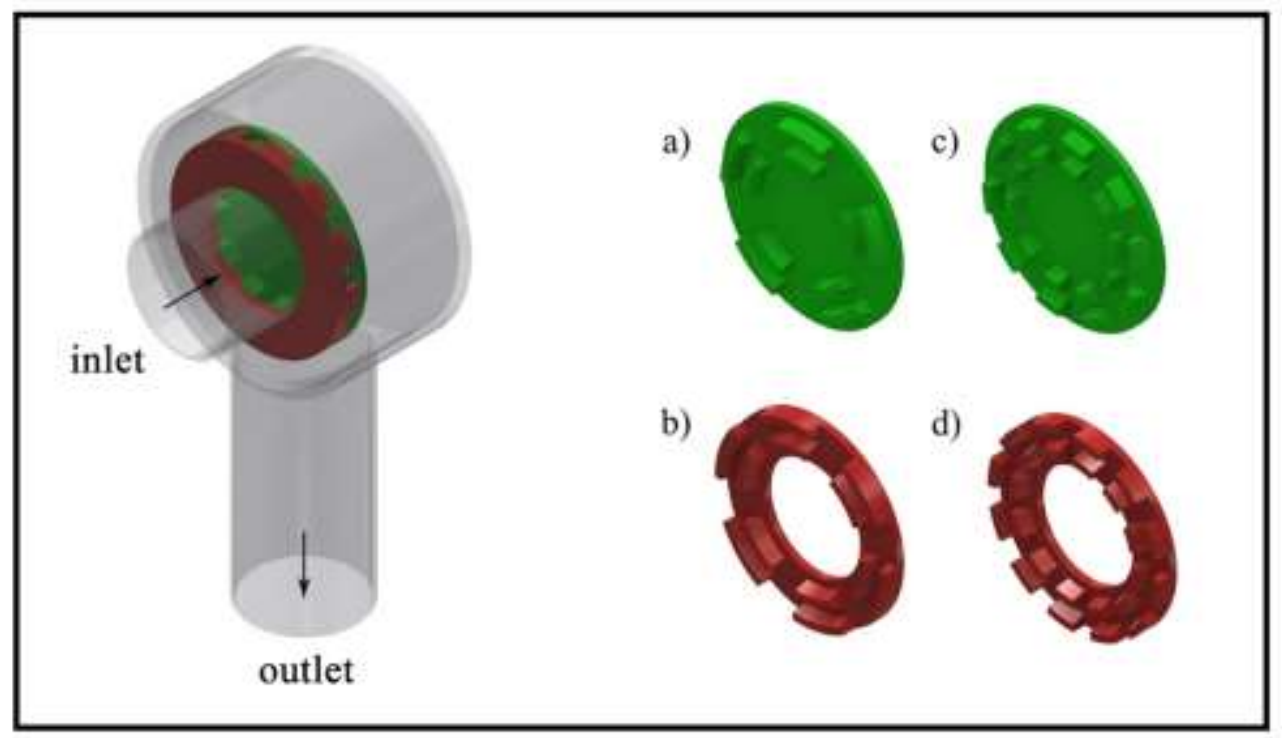

Fig. 1: Two variants of rotor and stator.

\section{2. Liquid}

Simulations were completed with single faze liquidated on viscose. Viscose is material used to produce art, silk, cellophane, rayon etc. This material shows non-Newtonian behaviour. Model was experimentally obtained, and it shows that liquid phase of viscose has a shear-thinning behaviour (Fig. 2).

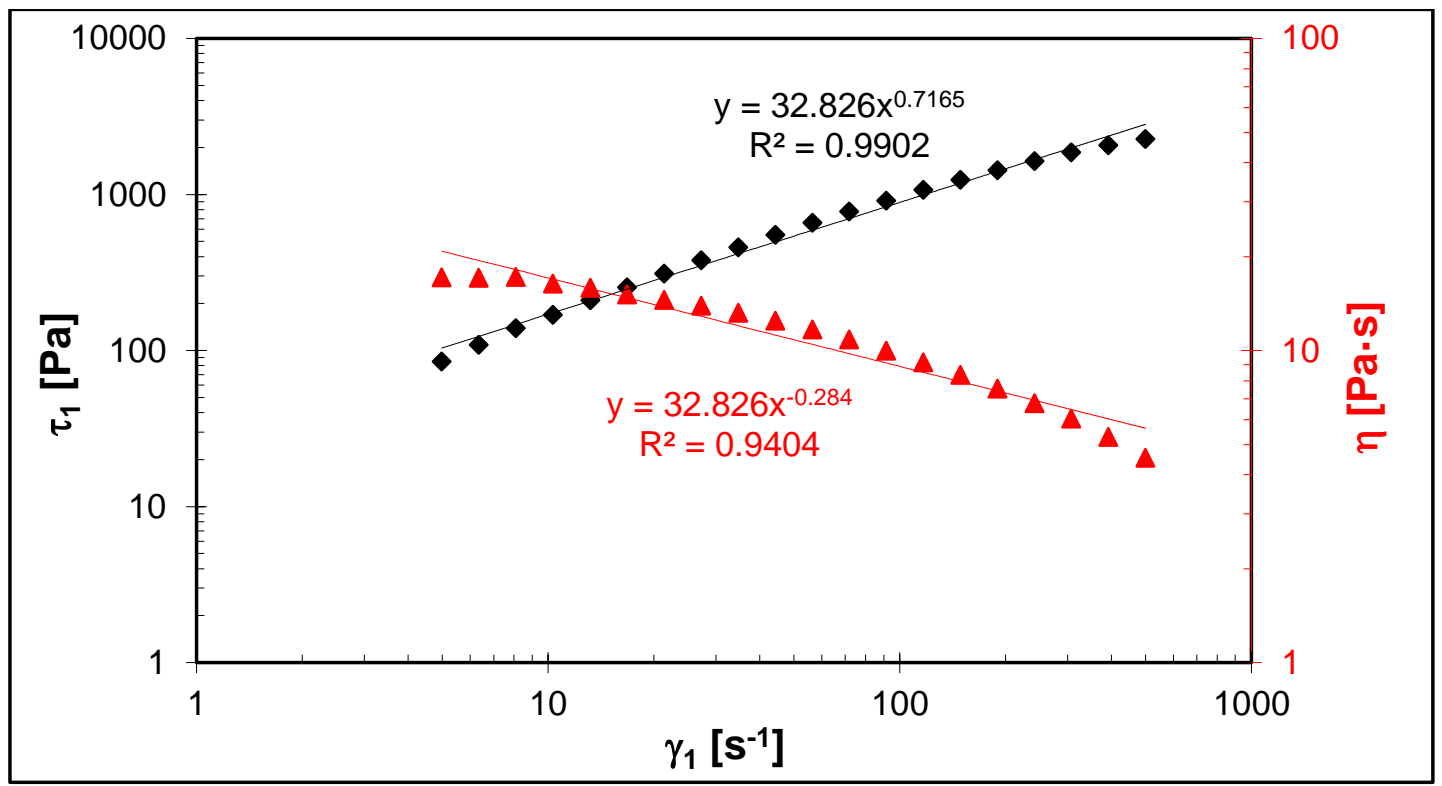

Fig. 2: Shear stress and apparent viscosity of used medium.

\section{3. Input parameters and method}

The computational greed for both geometries has about 2 mil. cells, most of them are in the rotor/stator region. Due to complicated geometry (blades on rotor and stator) the sliding mesh technique was used. Simulations were completed in 
laminar regime with the same boundary conditions for both geometries: $0.822 \mathrm{~m} / \mathrm{s}$ velocity inlet, 0 Pa pressure outlet and $2000 \mathrm{rpm}$ angular velocity.

\section{Results}

\section{1. Power consumption}

Power consumption was calculated from torque moment and angular velocity

$$
P=2 \pi n M
$$

In ANSYS Fluent software total moment is calculated as sum of pressure moment and viscous moment, so the power can be calculated as the sum of pressure and viscous (Tab. 1).

Table 1: Power consumption.

\begin{tabular}{|l|l|l|l|}
\hline & Total power & Pressure & Viscous \\
\hline Geometry 1 & $51 \mathrm{~kW}$ & $15 \mathrm{~kW}$ & $36 \mathrm{~kW}$ \\
\hline Geometry 2 & $54.5 \mathrm{~kW}$ & $18 \mathrm{~kW}$ & $36.5 \mathrm{~kW}$ \\
\hline
\end{tabular}

Results of calculation show that total power consumption is almost equal for both variants. The second geometry needs more pressure part of power which can be explained by narrower slots in the stator. The viscous part of power consumption is equal for both cases. During tests on real equipment with similar geometry and liquid the similar power consumption was calculated.

\section{2. Velocity field}

Fig. 3 displays the velocity magnitude field in the mixers. The first geometry has a greater velocity outside of rotorstator region and fluid flow is more tangential. In opposite the second geometry velocity field shows more radial flow in the non-mixing region.

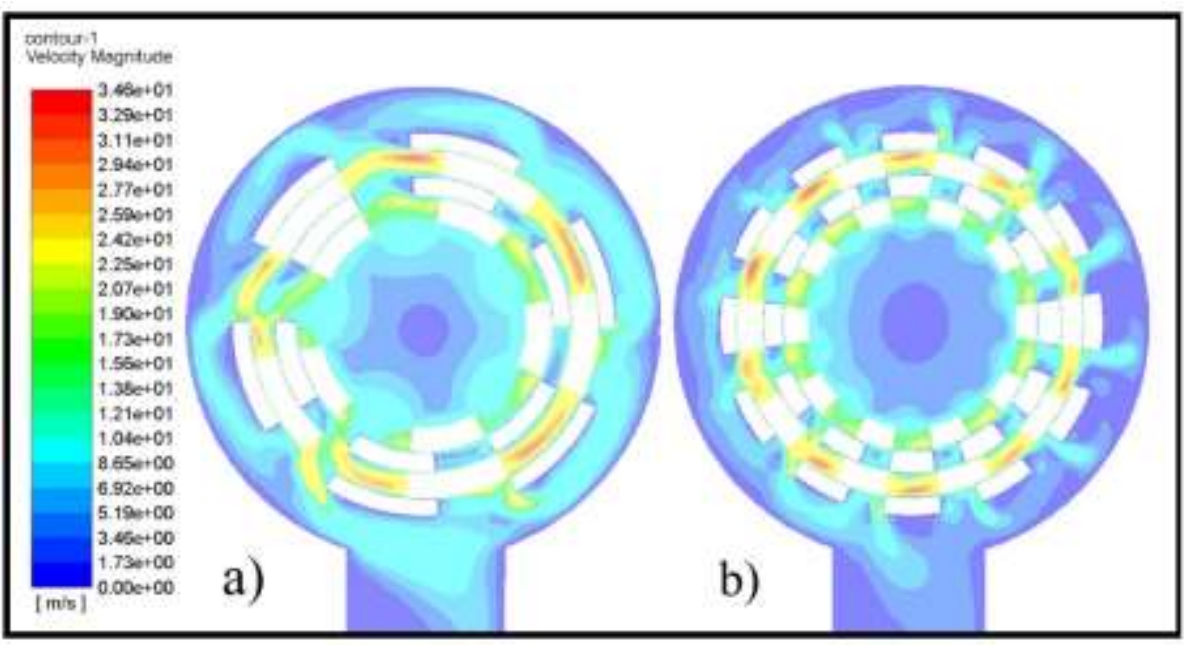

Fig. 3: Comparison of velocity magnitude fields.

\section{3. Velocity field in the hole}

Upon close examination of the nature of the current in the slot, it becomes clear that in the first case there are no abrupt changes in the direction of fluid flow. At the beginning of the movement of the rotor blade (Fig. 4a), the velocity vector 
slightly deviates from the tangential direction. When the rotor blade approaches the slot centre (Fig. 4b), the main fluid flow due to small changes in the direction of rotation of the rotor is directed from the mixing region, while only a small part of the current is in contact with the stator blade. When approaching the end of the slot (Fig. 4c), the remaining current has full contact with the surface of the stator blade and changes its direction to radial.

In the second case, due to the small width of the slot, the current flow does not have time to smoothly change its direction, and this already at the beginning of the movement of the rotor blade (Fig. 4d) radial direction of prevails. In the middle of the rotor blade (Fig. 4e), the flow does not change its direction but only increases its speed along the edge of the stator blade, which returns until the rotor blade completely closes the slot (Fig. 4f).
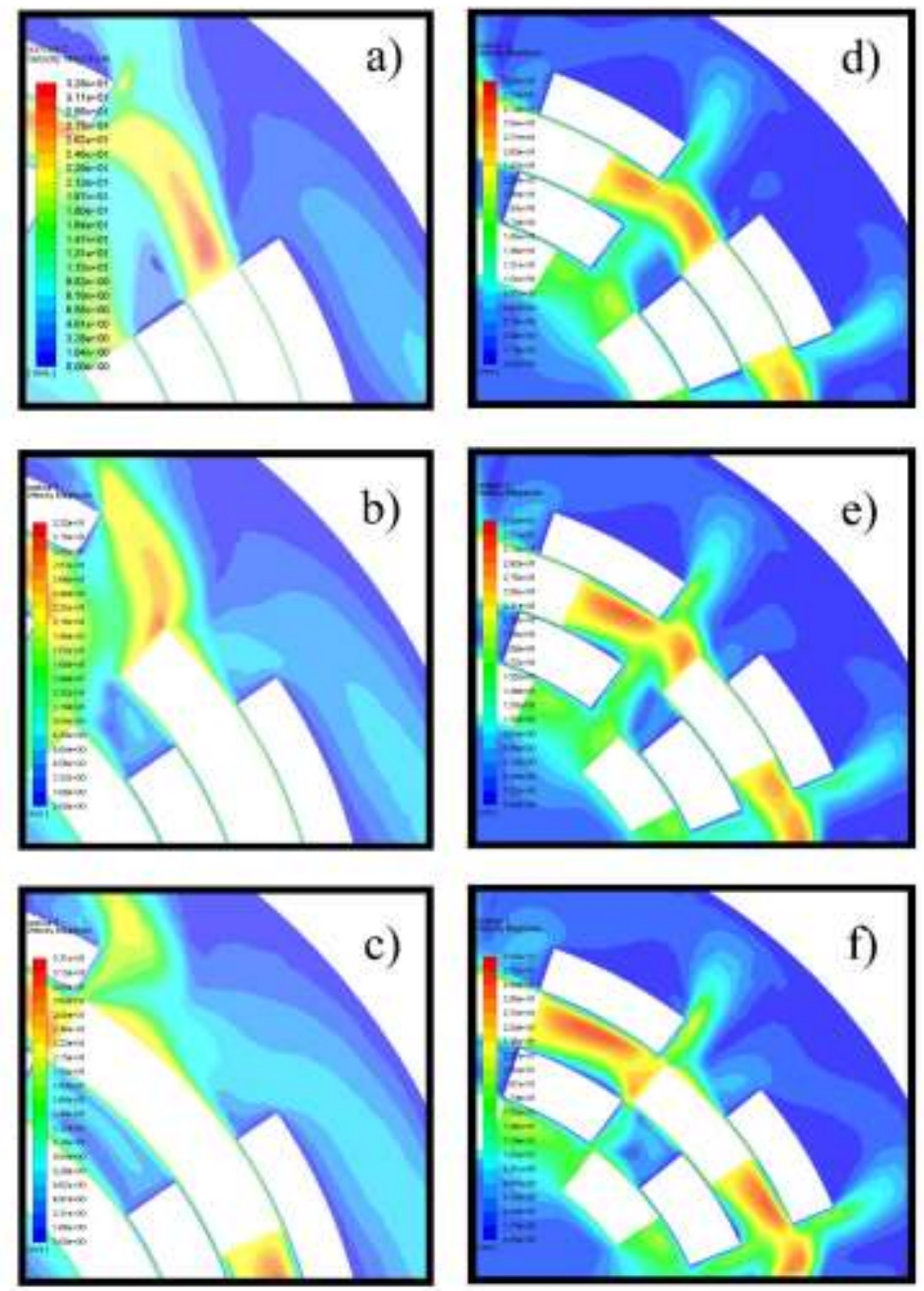

Fig. 4: Velocity magnitude field in the slot.

\section{4. Strain rate}

After velocity field the strain rate was examined. Both variants shows the same mechanism of strain rate changes. When rotor blade begins to pass through stator slot the strain rate peak appears on the bottom edge of stator blade. This peak disappears after rotor blade passes the half of the stator slot. AS rotor blade continues to move towards end of stator slot, the peak of strain rate occurs at the upper edge of the rotor blade and disappears when rotor starts to go between stator blades (Fig. 5). The strain rate magnitude between rotor and stator blades is the same for both variants. Considering the ratio of 
blades/slots between two geometries the second variant has two times more peaks of the strain rate which can lead to better dispergation results.
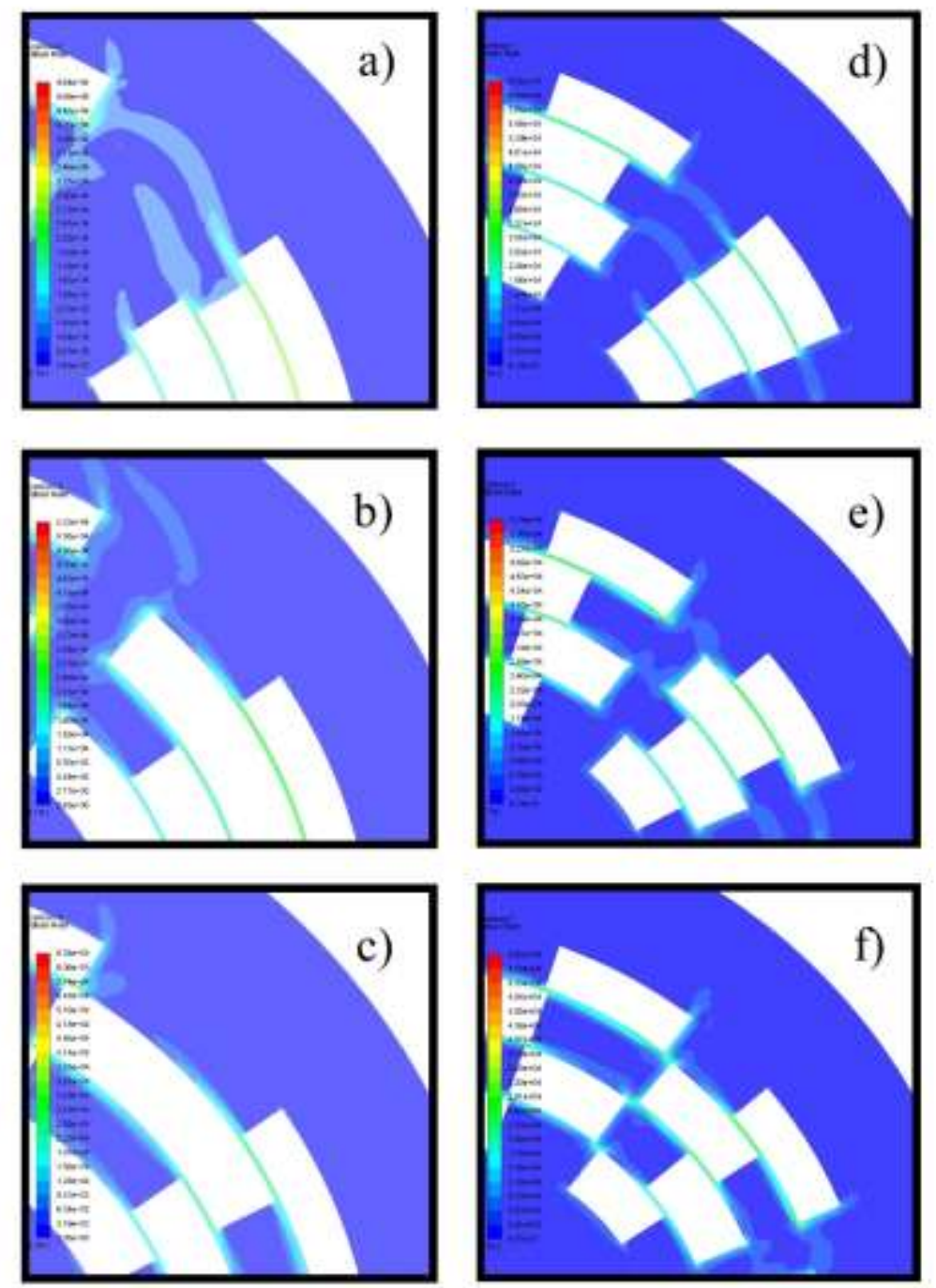

\section{Conclusion}

Fig. 5: Strain rate field in the slot.

The results obtained from simulations shows that in second case power consumption was increased a little by pressure loss part due to narrower slots. Velocity magnitude field shows that the first geometry has a better performance and fluid has a smooth change of direction from the hole. However, the second geometry shows better results at strain rate mean values and has twice more strain rate's peak (due to number of segments/slots). The results of the strain rate field assume, that the second geometry is more usable for dispergation based on pure strain. The influence of elongation still needs to be investigated.

Since results was obtained from simulations only there might be possible errors influenced by next parameters. Mesh quality can affect the results of simulation. The whole case was completed for laminar regime and there was no need to make near-wall mesh layers for better velocity profile. However, the mesh in the gap between rotor and stator segment might be not good even for laminar velocity profile. The second possible error is that apparent viscosity was set as undependable on 
temperature. Third possible error - initial simulation was completed for zero rpm and the velocity speed was slowly increased up to $2000 \mathrm{rpm}$. There is no information about how precisely the angular speed must be increased for better results.

\section{Acknowledgements}

This work was supported by the Grant Agency of the Czech Technical University in Prague, grant SGS $18 / 129 / \mathrm{OHK} 2 / 2 \mathrm{~T} / 12$

\section{References}

[1] V. A. Atiemo-Obeng, R. V. Calabrese, "Rotor-Stator Mixing Devices" in E. L. Paul, V. A. Atiemo-Obeng, S. M. Kresta, Handbook of Industrial Mixing, Wiley, Hoboken, 2004, pp. 479-505.

[2] H. H. Mortensen, F. Innings, A. Hakansson, "The effect of stator design on flowrate and velocity fields in a rotorstator mixer - An experimental investigation," Chemical Engineering Research and Design, vol. 121, pp. 245-254, 2017.

[3] H. H. Mortensen, F. Innings, A. Hakansson, "Local levels of dissipation rate of turbulent kinetic energy in a rotorstator mixer with different stator slot widths - An experimental investigation," Chemical Engineering Research and Design, vol. 130, pp. 52-62, 2018.

[4] A. Utomo, M. Baker, A. W. Pacek, "The effect of stator geometry on the flow pattern and energy dissipation rate in a rotor-stator mixer," Chemical Engineering Research and Design, vol. 87, pp. 533-542, 2009.

[5] G. I. Taylor, "The Formation of Emulsions in Definable Fields of Flow," Proc. Roy. Soc., vol. 29, pp. 71, 1879. 